\title{
Prueba de tamizaje simplificada para identificar a personas con visión disminuida en países en desarrollo ${ }^{1}$
}

\author{
J. E. Keeffe, ${ }^{2}$ J. E. Lovie-Kitchin, ${ }^{3}$ H. Maclean ${ }^{4}$ y H. R. Taylor $^{3}$
}

RESUMEN Se han creado pruebas sencillas pero útiles para el tamizaje de personas con visión disminuida en países en desarrollo. Estas pruebas, que miden la visión de lejos y de cerca y que se basan en el uso de una figura en E, fueron evaluadas y respaldadas mediante ensayos en que participaron personas de 4 a 90 años de edad, y se han puesto a prueba en los servicios de salud, educación y rehabilitación de 32 países en desarrollo. Se han calculado su sensibilidad y especificidad como instrumentos para la detección de la visión disminuida: en la prueba de visión a distancia, la sensibilidad es de 85\% y la especificidad, de 96\%; en la prueba de visión cercana, la sensibilidad es de $100 \%$ y la especificidad, de $84 \%$. Se ha demostrado que el contenido y el formato de las pruebas son adecuados para países en desarrollo y se ha comprobado la utilidad de las mismas para el tamizaje de la visión disminuida.

Alrededor de $90 \%$ de las personas con visión disminuida que existen en el mundo se encuentran en países en desarrollo (1). La provisión de pruebas adecuadas para estas zonas, donde hay escasez de personal adiestrado para atender a la salud ocular y pocos recursos, resulta por lo tanto imprescindible. Por ejemplo, en el África existe, en promedio, un oftalmólogo por cada millón de habitantes (2); pese

1 Se publicó en inglés en el Bulletin of the World Health Organization, Vol. 74, No. 5, 1996, con el título "A simplified screening test for identifying people with low vision in developing countries". (c) Organización Mundial de la Salud, 1996.

2 Universidad de Melbourne, Departamento de Oftalmología, Melbourne, Australia. Las solicitudes de separatas se deben enviar a este autor a la siguiente dirección postal: Royal Victorian Eye and Ear Hospital, 32 Gisborne Street, East Melbourne 3002, Australia.

3 Universidad Tecnológica de Queensland, Brisbane, Australia.

4 Universidad de Melbourne, Departamento de Oftalmología, Melbourne, Australia. a las iniciativas que actualmente se concentran en proveer adiestramiento, sigue habiendo una enorme escasez de personal con conocimientos especializados en el cuidado de la salud ocular y en la prevención de la ceguera.

Si es o no apropiada una prueba de agudeza visual se determina según sus características tecnológicas, su contenido y el adiestramiento necesario para saber administrarla. Para someter a un tamizaje de agudeza visual a un gran número de personas en un país en desarrollo a menudo es necesario reclutar a individuos de la comunidad que no han tenido adiestramiento. Por consiguiente, las pruebas que administran deben ser fáciles de aprender y de usar y sus resultados, fáciles de interpretar. Los materiales con que se administra la prueba deben ser duraderos y transportarse con facilidad. Además, la prueba adoptada no debe estar ligada a un contexto cultural en particular ni depender del nivel de alfabetismo, de tal manera que se pueda aplicar en cualquier parte del mundo.

El tamizaje con estas características sirve para detectar a aquellas personas con deterioro de la vista que necesiten ser remitidas a otro lugar para su tratamiento, y para identificar a las que tienen la vista potencialmente normal o alterada pero que han sido tratadas como si fueran ciegas. Por ejemplo, muchos estudiantes que asisten a programas y escuelas para ciegos en países en desarrollo tienen cierto uso de la vista y podrían llegar a leer materiales impresos, pero se les ha tratado como si fueran ciegos debido a que tienen algún grado de alteración visual. En África Oriental, hasta 80\% de los estudiantes clasificados de ciegos e inscritos en escuelas para ciegos o en programas especiales para personas con visión disminuida tenían mala agudeza visual que no había sido detec- 
tada con anterioridad. Muchos recibían la enseñanza mediante métodos no visuales, como el Braille (J. Keeffe y G. Nyaga, comunicación personal).

Nuestro estuche para evaluar la disminución de la vista en países en desarrollo contiene una ficha para la prueba de agudeza visual, una máscara agujereada para detectar alteraciones de la refracción y dos manuales con instrucciones para la medición de la agudeza visual, la evaluación de la visión funcional e información general sobre los efectos de la visión disminuida (3). Las pruebas se pueden aplicar en niños (si tienen más de 5 años aproximadamente) y adultos. El presente artículo describe la creación y validación de estas pruebas que miden la capacidad para ver de lejos y de cerca.

\section{MATERIALES Y MÉTODOS}

La ficha que se adoptó para hacer la prueba fue similar a la que había creado y puesto a disponibilidad la OMS, en la que se usaban optotipos de dos tamaños distintos sobre una pequeña tarjeta plástica cuadrada. Se usó la figura E en diferentes orientaciones, destinada a personas analfabetas, o los anillos de Landolt. La nueva ficha plástica para evaluar la agudeza visual mide $37 \mathrm{~cm} \times 18,5 \mathrm{~cm}$; cuando se dobla por la mitad, el cuadrado que se produce tiene en las caras externas optotipos que permiten medir la capacidad para ver de lejos. En la parte interna, la prueba que evalúa la capacidad para ver de cerca está impresa de un lado y las instrucciones sobre el uso de ambas pruebas, del otro (figura 1).

\section{El tamizaje de la capacidad para ver de lejos}

El propósito de la prueba que evalúa la capacidad para ver de lejos es detectar casos de visión normal o disminuida, y no obtener una medición precisa de la agudeza visual a distancia. Por lo tanto, las diferencias entre pruebas (uso de una sola letra o de un conjunto de cuatro letras) y la consiguiente variación en la interacción con el con-
FIGURA 1. Ficha para Medir la Agudeza Visual. El panel superior muestra los optotipos que aparecen en la cara externa de la ficha cuando está doblada por la mitad. Los tamaños tipográficos usados para medir la visión cercana y las instrucciones que aparecen en las caras internas se ilustran en el panel inferior
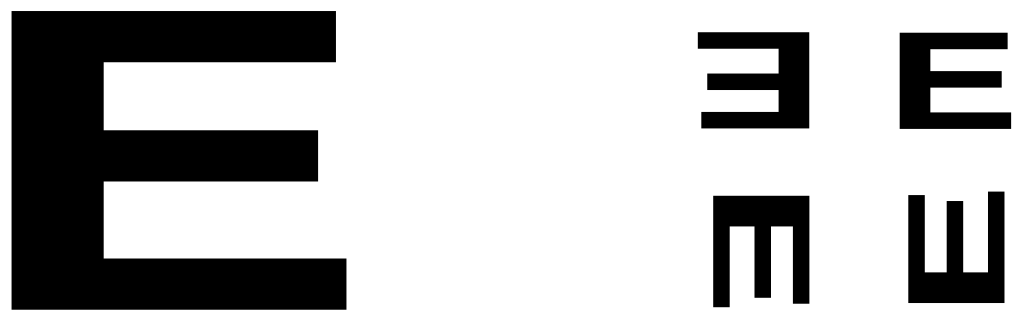

VISIÓN A CORTA DISTANCIA

Indique a la persona, acercándose a ella, cómo hacer la prueba de la $\mathrm{E}$. Indíquele la orientación de las Es. Investigue la visión a corta y larga distancia para determinar cuál es la $\mathrm{E}$ más pequeña que la persona puede identificar.

\section{VISIÓN A LARGA DISTANCIA}

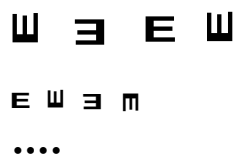

1. Haga la prueba con las 4 Es pequeñas a una distancia de 6 metros. La visión es normal (de 6/18 o Si no se pueden ver como aun mejor) si por lo menos 3 de mínimo 3 de las Es más las 4 Es pequeñas se pueden pequeñas, haga la prueba con ver. Si se pueden ver las Es más pequeñas en la prueba de visión cercana, no es necesario continuar con más pruebas. la E grande.

2. Haga la prueba con la $\mathrm{E}$ grande a una distancia de 6 metros. Rote la ficha para orientar la $E$ en cuatro direcciones distintas. Si se pueden leer las Es, la visión Si no se pueden leer como es de 6/60. Haga la prueba usando mínimo 3 de las Es grandes, el agujero. Si la visión mejora, haga la prueba a 3 metros de remita a la persona para que le distancia. hagan anteojos; si no mejora, envíela al oculista.

3. Haga la prueba con la $\mathrm{E}$ grande a 3 metros de distancia. Rote la ficha para orientar la $E$ en cuatro direcciones distintas. Si se pueden leer como mínimo 3 Si no se pueden ver como de las 4 Es, la visión es de 3/60. mínimo 3 de las Es grandes, Repita la prueba con el agujero haga la prueba con el agujero y para el número 2. remita al paciente al oculista para explorar la posibilidad de un tratamiento.

VISIÓN A CORTA DISTANCIA

4. Permita que la persona sostenga en la mano la ficha a la distancia que desee. Haga la prueba empezando con las Es más grandes y prosiguiendo con las más pequeñas. Por lo menos 3 de las 4 deben identificarse correctamente en cada línea antes de seguir al próximo renglón. Si solamente se pueden ver las letras más grandes (N48), averigüe si se consigue mejoría con el uso de lentes de aumento y remita al paciente al oculista para que se investigue la posibilidad de un tratamiento. La letra de tamaño mediano (N20) es similar a la que se usa para libros impresos en letra grande. La letra más pequeña (N8) es similar a la que se usa para libros y revistas.

Si la ficha para investigar la visión a corta distancia se usa a una distancia de 6 metros y se pueden leer las Es grandes, la visión es de 6/6 (normal).

Los procedimientos que deben seguirse para hacer la prueba están descritos en el Libro 1 - Tamizaje para detectar disminución de la visión. Prueba de agudeza visual. 
torno no se consideran desventajas. Se entiende, sin embargo, que para poder obtener una medición precisa de la agudeza visual sería necesario controlar estos factores $(4,5)$. Se recomienda (véanse las instrucciones que vienen con la prueba, figura 1) que se mida la visión a distancia con un mínimo de cuatro optotipos, independientemente del estado de la visión. Se ha adoptado como criterio para pasar la prueba, sea cual sea el estado de la visión, el poder identificar correctamente tres de cada cuatro Es. Se mide primero la visión a distancia con el juego de cuatro figuras E, que miden $18 \mathrm{~m}$. Si se identifican correctamente tres de las cuatro figuras a una distancia de 6 metros, la visión se clasifica de "normal", es decir, 6/18 o más, y no hace falta ninguna prueba adicional para medir la visión a distancia. Si no se identifican correctamente tres de las cuatro figuras, la E más grande (de $60 \mathrm{~m}$ ) se presenta en cuatro orientaciones diferentes. Si se identifican correctamente tres de las cuatro presentaciones, la persona sufre un deterioro visual equivalente a 6/60, o menos grave. Si no se identifican correctamente, la prueba debe repetirse a una distancia de 3 metros.

Independientemente del resultado obtenido con la letra E grande, se recomienda que se vuelva a evaluar la visión usando un agujero diminuto. $\mathrm{Si}$ la misma mejora, puede que haya un error de refracción importante $y$, de ser posible, la persona debe ser remitida para hacerse una corrección óptica. Si la visión no mejora, lo más probable es que haya una enfermedad ocular y el paciente debe ser remitido, donde haya servicios adecuados, para que se investigue la enfermedad y se le dé un posible tratamiento. Si se rota la ficha, los símbolos pueden orientarse en diferentes direcciones para evitar que se den respuestas de memoria al hacerse la prueba en ambos ojos, en un solo ojo y con el agujero.

\section{El tamizaje de la capacidad para ver de cerca}

Para vencer diferencias de carácter lingüístico o de alfabetismo dentro de un mismo país y entre distintos países, el símbolo E también se adoptó para investigar la visión cercana. Esta prueba contiene optotipos de tres tamaños, en vez de los seis o más que habitualmente se usan. Se adoptó el sistema puntual para designar el tamaño de la letra $(6,7)$. La primera versión de la prueba utilizaba dos tamaños, N8 y N20. Los comentarios recibidos después de aplicar la prueba indicaron que también se necesitaba un tamaño más grande para identificar a las personas que tenían la visión alterada pero funcional, de manera que se añadió el tamaño N48. En el cuadro 1 se indican los tamaños de las letras en puntos, unidades logMAR y unidades $M$.

Como la altura exacta de las letras varía según los distintos tipos, se midió la altura de las letras en numerosas pruebas para establecer una medida común o promedio para cada uno de los tamaños de letra elegidos. La E correspondiente a cada tamaño equivale en altura a la del cuerpo de la letra minúscula, es decir, la altura de la " $\mathrm{e}$ " o del componente circular de la " $\mathrm{d}$ ". La E se configura según el principio de Snellen en cuanto a su altura, anchura y la distancia entre espacios (8). La interacción con el contorno se controla procurando que la distancia entre las Es sea igual a la anchura de la E que aparece en esa línea, y que el espaciamiento entre líneas sea igual a la altura de las Es que aparecen en la línea de encima.

Los optotipos más pequeños (N8) se aproximan al tamaño tipográfico que se usa habitualmente para libros y periódicos. Las Es de tamaño N20 se asemejan al tipo empleado en libros

CUADRO 1. Tamaños tipográficos equivalentes en puntos, en el sistema métrico decimal y en el sistema logMAR

\begin{tabular}{lcc}
\hline Puntos & Unidades M $^{\mathrm{a}}$ & LogMAR $^{\mathrm{b}}$ \\
\hline N8 & $1 \mathrm{M}$ & 0.6 \\
N20 & $2.5 \mathrm{M}$ & 1.0 \\
N48 & $6 \mathrm{M}$ & 1.4 \\
\hline
\end{tabular}

a Referencia 5.
b Referencia 7. especiales escritos en letra grande. El tamaño más grande se aproxima al de las letras que se usan en titulares, rótulos o carteleras. También corresponde al tamaño del optotipo 6/6. Cabe advertir, por lo tanto, que si se identifica correctamente la línea de tamaño N48 a una distancia de 6 metros, la agudeza visual a distancia es 6/6.

El propósito de la prueba es dar una medición de la visión funcional aplicable a tareas que se hacen de cerca mediante la estimación del tamaño de letra limítrofe, sin medir la agudeza de la visión cercana en sí misma, de tal modo que no hace falta una distancia estándar para efectuar la prueba. La ficha con que se hace la prueba puede sostenerse a cualquier distancia que le permita a cada persona ver óptimamente de cerca, y la distancia usada para hacer la prueba debe registrarse. Cabe recalcar que los niños pueden sostener los objetos muy cerca de los ojos para obtener una magnificación natural o relativa en función de la distancia. A la persona que se somete a la prueba se le pide que indique la dirección en que están orientadas las Es, empezando con los símbolos más grandes. Nuevamente, el criterio para pasar la prueba es poder identificar correctamente tres de cada cuatro símbolos. Si se identifican correctamente los símbolos más pequeños (N8), la visión cercana se considera normal en términos funcionales. Si se identifica correctamente el tamaño mediano (N20), se trata de personas que podrían usar la vista, aun en su estado disminuido, pero que deben ser remitidas a un examen médico, oftalmológico, o ambos. Si solo se identifican los símbolos más grandes (N48), los aparatos de aumento pueden ser beneficiosos y debe remitirse al paciente a donde pueda hacerse pruebas adicionales.

\section{La aplicación experimental de las pruebas en países en desarrollo}

Cuatro series separadas de pruebas sobre el terreno fueron efectuadas en un período de 3 años por trabajadores de la salud, educadores y trabajadores comunitarios dedicados a actividades 
de rehabilitación en 32 países de la zona pacífica, Asia, África o Europa oriental, con el fin de determinar si los materiales dieron resultados satisfactorios y llenaron las necesidades de los distintos contextos. La ficha con que se efectúa la prueba, los libretos que la acompañan y las planillas de evaluación se enviaron por correo a investigadores en países en desarrollo que necesitaban examinar la visión de personas inscritas en sus programas o efectuar un tamizaje visual. La administración de la ficha con que se efectúa la prueba por usuarios sin adiestramiento fue observada (por el primer autor) con el fin de determinar si se estaban siguiendo los procedimientos adecuados. Este autor usó la prueba en programas de atención ocular y en centros comunitarios de rehabilitación y educación en las Filipinas, Fiji, la India, Kenya y Uganda. La versión final de la ficha usada para hacer la prueba se aplicó para evaluar a 127 estudiantes de 5 a 20 años que asistían a escuelas para ciegos en Kenya y en Uganda. Estos estudiantes formaron parte de la misma muestra evaluada por Silver et al. (9), salvo que se excluyó a las personas que no podían percibir la luz. Los resultados de los tamizajes se compararon con los resultados generales que Silver et al. (9) obtuvieron al aplicar pruebas de ji cuadrado.

Se llenaron formularios de evaluación después de cada conjunto de pruebas sobre el terreno. Entre los datos recolectados figuraron si se habían o no entendido y seguido bien las instrucciones y qué optotipo (el anillo de Landolt o la E) se había preferido. Se hicieron los cambios apropiados entre las diferentes series de pruebas sobre el terreno.

\section{La validación}

La sensibilidad y especificidad de la prueba usada para investigar la visión disminuida se evaluaron y compararon mediante el uso de pruebas oculares convencionales. La sensibilidad de una prueba (i.e., el porcentaje de casos adecuadamente remitidos) indica la probabilidad de que la prueba identifi- que correctamente a las personas con visión normal.

Debido a la falta de instalaciones donde evaluar adecuadamente la agudeza visual a larga y corta distancia durante los ensayos sobre el terreno, razón por la cual se hacen necesarias estas pruebas de tamizaje, las pruebas se validaron en el Royal Victorian Eye and Ear Hospital en Melbourne. Se obtuvo el consentimiento libre y fundamentado de todos los participantes. Las pruebas para la medición de la visión a corta y larga distancia fueron administradas por un examinador vendado a 125 pacientes de 4 a 90 años según el orden consecutivo en que acudieron a las clínicas ambulatorias del hospital. En la muestra había personas con albinismo, alteraciones maculares relacionadas con la edad, cataratas, afaquia o seudofaquia y trastornos de la refracción. Muchas no tenían problemas oculares de importancia. Se sometieron a prueba la visión óptima a larga y corta distancia, tras corrección, en ambos ojos según las instrucciones proporcionadas para las pruebas oculares con objeto de reproducir la forma en que se efectuaba la prueba sobre el terreno. Para fines de comparación se usaron las carteleras tradicionales de Snellen a base de formas en E para medir la agudeza visual a distancia, y la prueba a base de la identificación de letras idénticas de Sheridan Gardiner (SG) o, para tamaños mayores de $18 \mathrm{~N}$, el libreto de selección de letras idénticas que acompaña a la Prueba de Visión Cercana para Niños (Near Vision Test for Children, o NVTC) $)^{5}$ para la agudeza visual a corta distancia. Las agudezas visuales que se midieron con estas pruebas convencionales se recogieron como parte de las pruebas clínicas de rutina efectuadas el mismo día por otro examinador.

No es posible conseguir una correspondencia exacta, punto por punto, entre las agudezas visuales medidas con las carteleras convencionales y con las pruebas de tamizaje; tampoco hace falta, si se considera la finalidad del

\footnotetext{
5 Publicado por Options Australia, Canterbury, Vic toria, Australia.
}

tamizaje. Las mediciones de la agudeza visual obtenidas con la prueba de Snellen, la de SG y la NVTC se agruparon a manera de ilustrar las mediciones equivalentes obtenidas con las pruebas nuevas (cuadros 2 y 3). Se dan las categorías de la OMS para cada grupo de medidas de agudeza visual a distancia.

\section{RESULTADOS}

Las nuevas pruebas de visión a distancia larga y corta dieron buenos resultados tanto en países en desarrollo como en las clínicas ambulatorias de hospitales en Australia, a las que acudieron niños desde los 4 años y personas que no hablaban el idioma del examinador. La falta de un mismo idioma no constituyó un obstáculo importante porque la prueba se podía explicar con demostración.

De los 127 estudiantes que asistían a escuelas para ciegos en Kenya y Uganda, más de $60 \%$ se clasificaron en la categoría de visión disminuida sobre la base de los resultados de la prueba de visión a distancia (cuadro 4 ), a pesar de que a la mayoría de los estudiantes se les impartía la enseñanza con métodos no visuales, como el Braille. El número de estudiantes en cada categoría de agudeza visual no fue significativamente diferente del obtenido en una población de características similares estudiada por Silver et al. (9) (prueba de ji cuadrado = 3,1; $P=0,2)$. La prueba de visión cercana reveló que 61 (49\%) de los estudiantes tenían una visión a corta distancia dentro de los límites normales y que otros 43 (34\%) podían leer materiales escritos con letras grandes o de tamaño normal si usaban lentes de aumento (cuadro 4).

La validación. Los dos sujetos (de un total de 125) que no pudieron terminar de hacerse las pruebas de validación en el Royal Victorian Eye and Ear Hospital fueron niños pequeños con discapacidad intelectual además de visión disminuida. Por consiguiente, solo se dispuso de los resultados de las prue- 
CUADRO 2. Categorías de alteración visual de la OMS y agudezas visuales que les corresponden en la nueva prueba de visión a distancia y en la tabla de Snellen

\begin{tabular}{lcc}
\hline Categoría de la OMS & Prueba nueva & Tabla de Snellen \\
\hline Visión "normal" & $6 / 6,6 / 18$ & $6 / 6,6 / 9,6 / 12,6 / 18$ \\
Visión disminuida & $6 / 60,3 / 60$ & $6 / 24,6 / 36,6 / 60,3 / 60$ \\
Ceguera & $<3 / 60$ & $<3 / 60$ \\
\hline
\end{tabular}

bas de tamizaje y de las pruebas de agudeza visual convencionales en el caso de 123 sujetos. De estos, $104(85 \%)$ tuvieron los mismos resultados en ambas pruebas de agudeza visual a larga distancia (figura 2). En total, 115 $(93 \%)$ fueron ubicados en la misma categoría de agudeza visual (es decir, con visión normal, con visión disminuida, o con ceguera) (cuadro 5).

CUADRO 3. Tres mediciones con la nueva prueba de visión a corta distancia y sus equivalentes con la prueba de SG (Sheridan Gardiner) y con la NVTC (Prueba de Visión Cercana para Niños)

\begin{tabular}{ll}
\hline Prueba nueva & \multicolumn{1}{c}{ SG o NVTC } \\
\hline N8 (normal) & $\mathrm{N} 5, \mathrm{~N} 6, \mathrm{~N} 8$ \\
$\mathrm{~N} 20$ (disminución & $\mathrm{N} 10, \mathrm{~N} 12, \mathrm{~N} 16, \mathrm{~N} 18, \mathrm{~N} 20$ \\
moderada) \\
$\begin{array}{c}\text { N48 (disminución } \\
\text { grave) }\end{array}$ & $\mathrm{N} 24, \mathrm{~N} 32, \mathrm{~N} 40, \mathrm{~N} 48$ \\
\hline
\end{tabular}

CUADRO 4. Resultados obtenidos con las pruebas de visión a larga y corta distancia en escuelas para ciegos en Kenya y Uganda $(n=127)$

\begin{tabular}{lc}
\hline & No. de estudiantes \\
\hline $\begin{array}{l}\text { Categorías de la OMS } \\
\text { Visión "normal" }(\geq 6 / 18)\end{array}$ & 14 \\
Visión disminuida & \\
$(<6 / 18$ a 3/60) & 78 \\
Ceguera $(<3 / 60)$ & 35 \\
Visión a corta distancia & \\
N8 & 61 \\
N20 & 43 \\
N48 & 9 \\
Sin respuesta $(>\mathrm{N} 48)$ & 14 \\
\hline
\end{tabular}

En cuatro sujetos $(3 \%)$ que no habrían sido detectados o remitidos adecuadamente si se hubiera usado la prueba de tamizaje se encontró que había visión disminuida según la prueba de agudeza visual de Snellen (resultados negativos falsos). Con la nueva prueba se detectó a otros cuatro $(3 \%)$ cuya agudeza visual era de $6 / 18$ o aun mejor (resultados positivos falsos). Por consiguiente, hubo un total de ocho $(6 \%)$ remisiones por error. La sensibilidad (porcentaje de remisiones correctas por deterioro visual o ceguera) fue de $85 \%$ y la especificidad (porcentaje con visión normal identificada correctamente y que no necesitaba remisión) fue de $96 \%$.

De los 77 sujetos cuya visión a corta distancia se midió, 69 (90\%) obtuvieron resultados similares con la nueva prueba de tamizaje y con las pruebas clínicas convencionales (cuadro 6). Todos los 42 sujetos que pudieron leer el tamaño N8 en la nueva prueba pudieron identificar o aparear letras de un
FIGURA 2. Distribución de los resultados de agudeza visual a larga distancia obtenidos con la prueba basada en la figura $E$ y con la prueba de Snellen. Las cifras correspondientes a cada coordenada indican el número de personas y los resultados obtenidos en la prueba respectiva. Por ejemplo, el punto cercano a la esquina inferior izquierda indica que 20 personas tuvieron una agudeza visual de $6 / 6$ en ambas pruebas

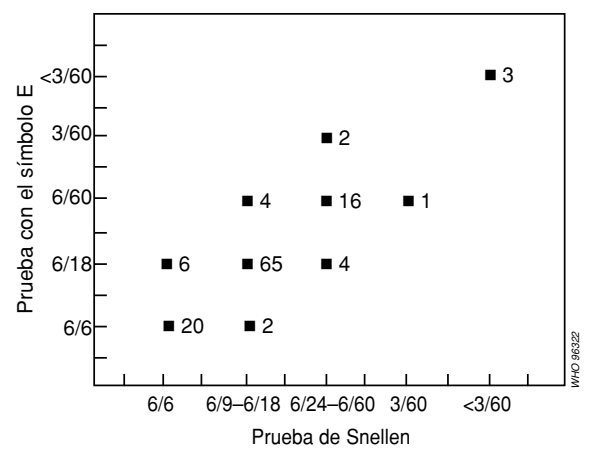

tamaño de N8 a N5 en la prueba de SG y en la NVTC. Ocho de los 35 sujetos que pudieron leer letras de tamaño N20 pero no N8 en la prueba nueva pudieron identificar letras más pequeñas entre N20 y N8 en las pruebas usadas para fines de comparación. Del total, $10 \%$ hubieran sido remitidos por "visión disminuida" sin que la tuvieran. La sensibilidad y especificidad de la prueba de visión a corta distancia fueron de $100 \%$ y $84 \%$, respectivamente (cuadro 6).

CUADRO 5. Comparación de los resultados obtenidos en la prueba simplificada de visión a corta distancia con los de la prueba de Snellen, en el Royal Victorian Eye and Ear Hospital. Las cifras horizontales se obtuvieron con la nueva prueba en $E$, es decir, en 4 y 19 sujetos se detectó visión disminuida. Las cifras verticales se obtuvieron con la prueba de Snellen. Tres sujetos se clasificaron de ciegos con ambas pruebas

\begin{tabular}{lccc}
\hline & \multicolumn{3}{c}{ Prueba de Snellen } \\
\cline { 2 - 4 } & Visión "normal" & Visión alterada & Ceguera \\
\hline Prueba simplificada & & & \\
Visión "normal" & 93 & 4 & 0 \\
Visión disminuida & 4 & 19 & 0 \\
Ceguera & 0 & 0 & 3 \\
& Sensibilidad & & Especificidad \\
& $85 \%$ & & $96 \%$ \\
\hline
\end{tabular}


CUADRO 6. Comparación de los resultados obtenidos con la nueva prueba de visión a corta distancia y con las pruebas convencionales (SG y NVTC) en el Royal Victorian Eye and Ear Hospital. Las cifras horizontales se obtuvieron con la nueva prueba basada en la figura $E$, es decir, en 8 y 27 sujetos se detectó visión disminuida. Las cifras verticales se obtuvieron con la prueba de SG y con la NVTC

\begin{tabular}{lcc}
\hline & \multicolumn{2}{c}{ Pruebas convencionales } \\
\cline { 2 - 3 } & Visión "normal" & Visión disminuida \\
\hline Prueba simplificada & 8 & 27 \\
Visión disminuida & 42 & 0 \\
Visión "normal" & Sensibilidad $100 \%$ & Especificidad $84 \%$ \\
\hline
\end{tabular}

\section{DISCUSIÓN}

Tamaño de los optotipos. El uso de optotipos de dos tamaños para medir la agudeza visual a larga distancia es adecuado para fines del tamizaje y para establecer información de base para evaluar la visión funcional. Cuando se aplica la prueba a 3 y 6 metros de distancia según se necesite, estos tamaños permiten clasificar la agudeza visual de acuerdo con las categorías generales de la OMS de visión "normal", visión disminuida, o ceguera (10).

La prueba de visión cercana se usa para determinar si hay visión funcional para tareas a corta distancia, el tamaño aproximado de la letra necesario para la lectura, y si es o no necesario remitir a la persona para la corrección de trastornos de la refracción o la prescripción de lentes para mejorar la vista. La visión cercana de los individuos que pueden identificar las Es más pequeñas podría clasificarse dentro de lo normal; la identificación de las letras más grandes solamente indica que hay uso de los ojos para ver de cerca pero una capacidad reducida para diferenciar los detalles más pequeños. Si el tamaño más grande no se puede leer incluso a una distancia muy corta, se debe contemplar el uso de métodos no visuales para la captación del lenguaje impreso y de otra información.

Los símbolos usados para la prueba. Otras pruebas para el tamizaje de la vista, que han sido elaboradas para personas que no saben leer en virtud de su edad o analfabetismo y para individuos con diversas discapacidades, son las fichas de Lighthouse (11), las figuras de Kay (12), las fichas de Allen (13) y los símbolos LH (14). Las figuras usadas en estas pruebas han sido elegidas porque son conocidas y fáciles de identificar (12). Las figuras a menudo representan objetos de uso común en países industrializados, pero no en países en desarrollo, donde plantea problemas la falta de familiaridad con las figuras en todos los estratos culturales. El problema es menos acentuado en el caso de las pruebas LH (14), ya que los símbolos más estilizados no representan objetos específicos.

Los símbolos para personas analfabetas (la cartelera a base de la letra E, el anillo de Landolt y la Mano de Sjögren) se han usado extensamente y se han recomendado para programas de tamizaje (13, 15-19). El uso de las figuras en E o del anillo de Landolt puede plantear dificultades en niños preescolares pequeños debido a la orientación de los símbolos en determinada dirección, de izquierda a derecha (13), pero ambas pruebas han sido usadas y recomendadas para niños de 5 años de edad en adelante $(11,14,19)$. La utilidad de la prueba a base del símbolo E dependerá de la edad de la persona examinada y de la finalidad del tamizaje. Weale ha elaborado un protocolo de tamizaje para que los maestros puedan identificar a los niños con un déficit de la visión que necesiten ser remitidos a un centro de atención ocular (19); el instrumento usado para el tamizaje es una cartelera con el símbolo
E en tres tamaños para medir la visión a larga distancia.

Es fácil explicar el procedimiento basado en la cartelera con el símbolo E y el que utiliza el anillo de Landolt, y las barreras lingüísticas son fáciles de vencer. También existe la ventaja de que una sola persona puede administrar la prueba. En nuestro proyecto, tanto la cartelera con la letra E como el anillo de Landolt se usaron para medir la visión a larga y corta distancia en países en desarrollo. Los comentarios recibidos de la mayoría de los examinadores en distintos países revelaron una preferencia por la cartelera con el símbolo E. Las pruebas de tamizaje en el terreno se aplicaron en su mayor parte en niños de edad escolar, aunque no exclusivamente.

La cartelera con el símbolo E, que no exige la lectura de números o letras, se adoptó para medir la visión a corta distancia. Esta prueba indica el grado de detalle que se puede percibir; no es una lectura y solamente sirve para determinar el tamaño de letra mínimo que se puede leer. Leer no es la única tarea que se efectúa de cerca $\mathrm{y}$, para muchas personas, no es ni siquiera una tarea importante.

Una ventaja de la cartelera con Es o del anillo de Landolt es que los símbolos pueden mostrarse repetidas veces; siguen siendo, aun en distintas orientaciones, objetos inteligibles y útiles para la prueba, porque la dirección de la E o el espacio en el contorno del anillo se pueden cambiar cada vez que se muestran las figuras. Cuando se usan objetos, cifras u otros símbolos, rotarlos de ese modo no es posible, de manera que se necesitan muchos optotipos para cada nivel de agudeza visual, o bien dos fichas distintas. El uso de fichas grandes o numerosas no es factible ni deseable para el trabajo de campo.

\section{La importancia del tema para países} en desarrollo. Las observaciones cualitativas de quienes han usado la ficha para hacer la prueba en países en desarrollo indican que el formato y el contenido se ajustan a las necesidades locales. La ficha con que se investiga la agudeza visual es apropiada, fácil de 
aprender y fácil de usar en diversas situaciones.

La prueba también tuvo un buen rendimiento en el estudio de validación. Ninguna de las personas con visión disminuida dejó de ser captada por las pruebas de visión a corta distancia y solamente $3 \%$ de las sometidas a la prueba de visión a larga distancia pasaron desapercibidas. Si se tiene en cuenta la sencillez de las pruebas, estas cifras son muy favorables. Schmidt, en una revisión de la exactitud de las técnicas de tamizaje visual empleadas en países desarrollados, encontró gran variación entre los materiales y procedimientos usados para el tamizaje (19). La proporción total de remisiones por equivocación fluctuó entre 0 y $58 \%$, pero los criterios de remisión fueron más rigurosos que los recomendados para países en desarrollo. La proporción de remisiones excesivas y la de remisiones insuficientes son inferiores a las detectadas en el presente estudio únicamente al incluirse el examen ocular como parte del tamizaje visual.

La diferencia de rendimiento entre nuestra prueba para la medición de la visión de cerca y las pruebas de SG y NVTC se podría deber al tipo de cartelera utilizada. En la prueba para investigar la visión a corta distancia se usaron tres símbolos espaciados uniformemente, en escalones logarítmicos parejos regidos en su tamaño por los criterios descritos por Bailey y Lovie (4) (figura 1). La discriminación e identificación de los optotipos en este formato encierran más dificultad que en el caso de las letras bien espaciadas o aisladas que se usan en la prueba de SG y en la NVTC.

La aplicación experimental de estas pruebas para la detección de la visión disminuida en la etapa en que las mis- mas fueron elaboradas ha servido para demostrar que son apropiadas para países en desarrollo. Dichas pruebas logran clasificar acertadamente a las personas según las categorías de visión "normal", visión disminuida, o ceguera. Sus resultados se pueden usar para decidir si remitir a las personas para que reciban tratamiento o corrección de los trastornos de la refracción y, tomados junto con los resultados de la evaluación de la visión funcional, pueden servir para evaluar cualquier disminución de la vista o para fines de rehabilitación. El presente estudio ha confirmado que estas pruebas que detectan si hay visión disminuida constituyen instrumentos cuyo uso es adecuado en las situaciones aquí descritas.

\section{REFERENCIAS}

1. Brohier WG. Setting the scene. En: Johnston AW, Lawrence $\mathrm{M}$, eds. Low vision ahead II. Melbourne, Australia: Association for the Blind; 1990. pp. 9-13.

2. Foster A, Johnson G. Blindness in the developing world. Br J Ophthalmol 1993;77: 398-399.

3. Keeffe JE, et al. Low vision assessment and training materials for use in developing countries. En: Koojiman AC, et al., eds. Low vision: research and new developments in rehabilitation. Amsterdam: IOS Press; 1994. pp. 47-50.

4. Bailey IL, Lovie JE. New design principles for visual acuity letter charts. Am J Optom Physiol Opt 1976;53:740-745.

5. Lovie-Kitchin JE. Validity and reliability of visual acuity measurements. Ophthalmic Physiol Opt 1988;8:363-370.

6. Law FW. Reading types. Br J Ophthalmol 1952;36:689-690.
7. Johnston AW. Making sense of the M, N, and $\log$ MAR systems of specifying visual acuity. Probl Optom 1991;3:394-407.

8. Sloan LL, Brown DJ. Reading cards for the selection of optical aids for the partially sighted. Am J Ophthalmol 1963;55:1187-1199.

9. Silver J, et al. Low vision in East African blind school students: need for optical low vision services. $\mathrm{Br}$ J Ophthalmol 1995;79: 814-820.

10. International classification of impairments, disabilities, and handicaps. Geneva: World Health Organization; 1980.

11. Faye EE. A new visual acuity test for partiallysighted non-readers. J Pediatr Ophthalmol 1968;5:210-212.

12. Kay H. New method of assesing visual acuity with pictures. Br J Ophthalmol 1983;67:131-133.

13. Fonda GE. Management of low vision. New York: Thieme-Stratton; 1981.
14. Duke-Elder S, ed. VII, System of ophthalmology. En: The foundations of ophthalmology. London: Kimpton; 1962.

15. Oliver N, Nawratzki I. Screening of preschool children for ocular anomalies. $\mathrm{Br} \mathrm{JOph}$ thalmol 1971;55:462-466.

16. Thylefors B. Vision screening of illiterate populations. Bull World Health Organ 1997; 55:115-119.

17. Taylor HR. Applying new design principles to the construction of an illiterate E chart. Am J Optom Physiol Opt 1978;55:348-351.

18. Weale RA. Screening children's eyesight: guide for schoolteachers. World Health Forum 1983;4:121-124

19. Schmidt PP. Vision screening. En: Rosenbloom AA, ed. Principles and practice of pediatric optometry. Philadelphia: Lippincott; 1990. pp. 467-485.
ABSTRACT

\section{A simplified screening test for identifying people with low vision in developing countries}

Simple but effective tests have been produced for screening subjects with low vision in developing countries. These tests of distance and near vision, based on the E test, were evaluated and validated in trials with people aged 4-90 years, and have been field tested in the health, education and rehabilitation services in 32 developing countries. Their sensitivity and specificity as screening tools for low vision have been calculated: sensitivity of $85 \%$ and specificity of $96 \%$ for the distance vision test, and sensitivity of $100 \%$ and specificity of $84 \%$ for the near vision test. The content and format of the tests have been demonstrated to be appropriate for developing countries, and their effectiveness for screening for low vision has been confirmed. 